\title{
Decompositions of binomial ideals
}

\author{
THOMAS KAHLE
}

\begin{abstract}
The Macaulay2 package Binomials contains implementations of specialized algorithms for binomial ideals, including primary decomposition into binomial ideals. The current implementation works in characteristic zero. Primary decomposition is restricted to binomial ideals with trivial coefficients to avoid computations over the algebraic numbers. The basic ideas of the algorithms go back to Eisenbud and Sturmfels' seminal paper on the subject. Two recent improvements of the algorithms are discussed and examples are presented.
\end{abstract}

BINOMIAL IDEALS. Let $S=\mathbb{k}\left[x_{1}, \ldots, x_{n}\right]$ denote the polynomial ring over a field $\mathbb{k}$. A binomial ideal $I \subset S$ is an ideal generated by binomials $x^{u}-\lambda x^{v}$, where $u, v \in \mathbb{N}^{n}$ are exponent vectors and $\lambda \in \mathbb{k}$ is a coefficient. Monomials are also considered binomials. Assumptions on $\mathbb{k}$ will be forced upon us when computing binomial primary decompositions. The ideal $\left\langle x^{3}-1\right\rangle$ has no primary decomposition into binomial ideals when $\mathbb{k}$ does not contain a third root of unity. Interest in binomial ideals is due to the frequency with which they arise in applications. To name one, in algebraic statistics one is interested in primary decompositions of conditional independence ideals whose components describe various combinatorial ways in which a set of conditional independence statements can be realized $\left[\mathrm{F}, \mathrm{HHH}^{+}\right]$. Because the minimal primes of binomial ideals are toric ideals [ES], binomial conditional independence models are unions of exponential families. In particular they are unirational. Knowledge of a primary decomposition also gives a piecewise parameterization of such models.

The Macaulay2 [M2] package Binomials offers specialized implementations of primary decomposition, radical computations and minimal and associated primes. The starting point for this implementation was $\S 9$ in Eisenbud and Sturmfels' foundational paper [ES], but various improvements have been discovered and implemented. Binomials is the fastest and often only way to compute large primary decompositions of binomial ideals. The following example demonstrates a few common operations.

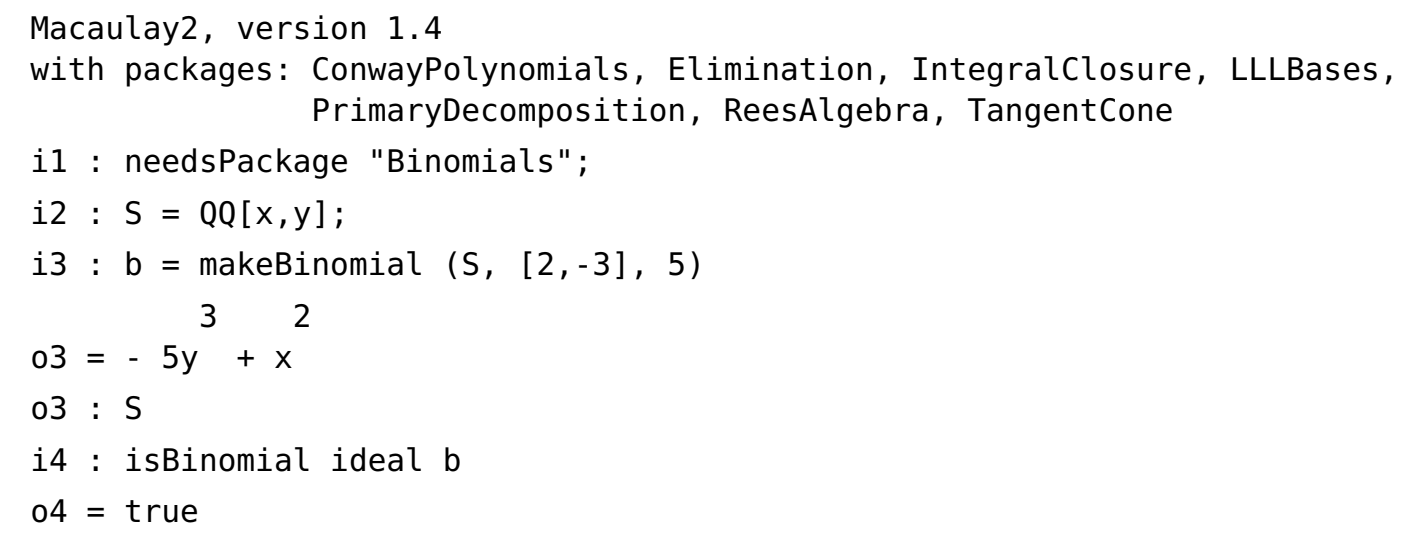

2010 Mathematics Subject Classification. 13P15, 13 C99.

Binomials version 1.0. 


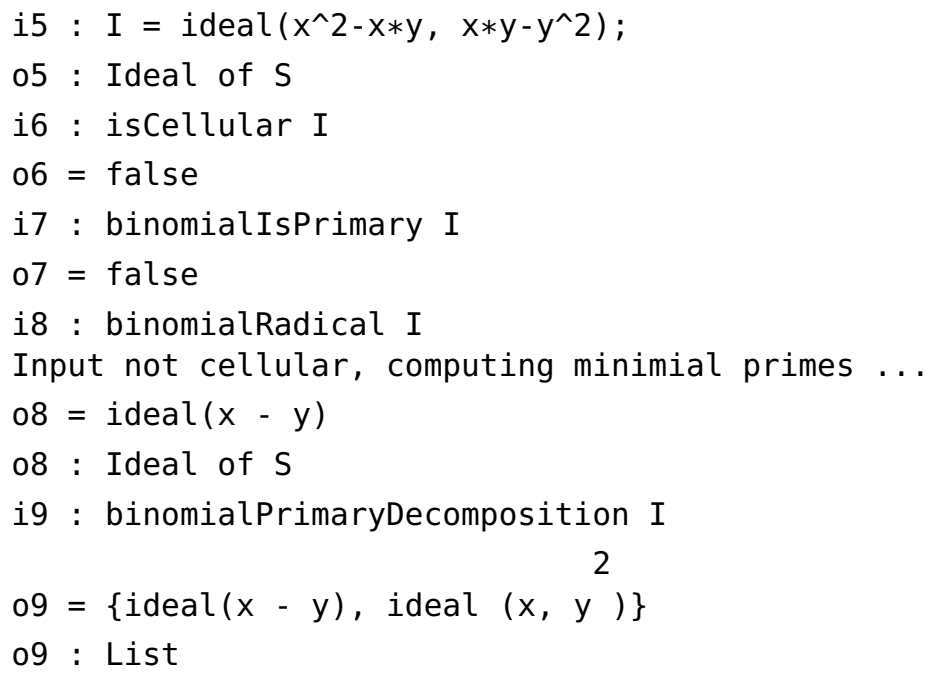

A binomial primary decomposition starts with a cellular decomposition. Recall that a binomial ideal $I \subset S$ is cellular if in $S / I$ every monomial is either regular (i.e. a nonzerodivisor) or nilpotent. The implemented algorithm to compute a cellular decomposition is discussed in [K, OS]. Since cellular decomposition is independent of $\mathbb{k}$, it can serve as a first approximation of primary decomposition over any field. In this paper, we focus on decomposing a cellular binomial ideal further. To this end, assume that $I$ is $J$-cellular for some $J \subset[n]$, that is, the variables with indices in $J$ are regular, while the variables with indices in $\bar{J}:=[n] \backslash J$ are nilpotent.

COMPUTING ASSOCIATED PRIMES. If $\mathbb{k}$ is algebraically closed, then the associated primes of a binomial ideal are guaranteed to be binomial. Since computer algebra systems usually do not implement algebraically closed fields, the input binomial ideals are restricted to be generated by unital binomials $x^{u}-x^{v}$. In this case, the binomial primary decomposition together with the associated primes exist over a cyclotomic extension of $\mathbb{Q}$, see $[\mathrm{K}]$. If necessary, Binomials will construct this extension and return its result over a different ring.

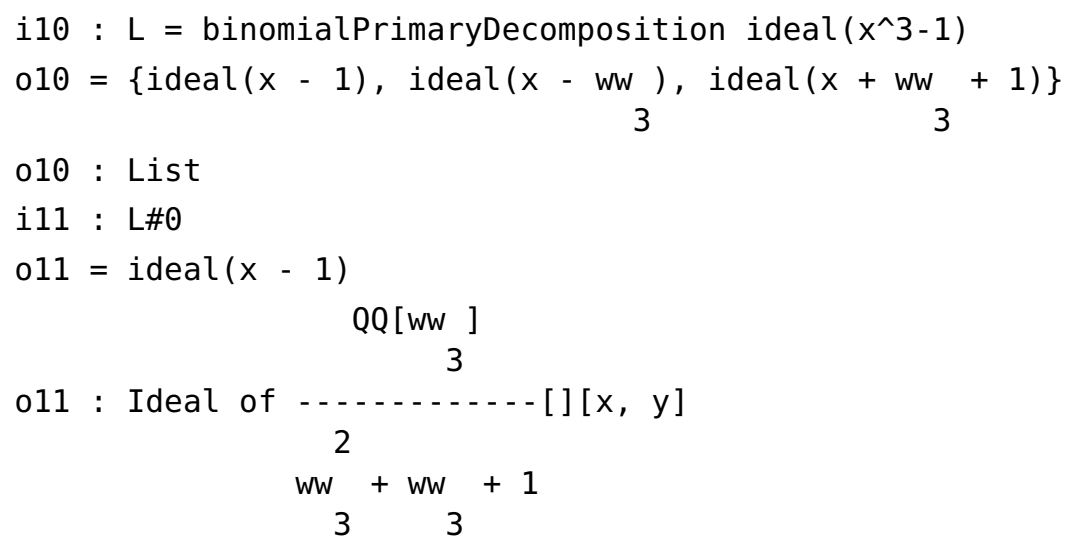

In the following discussion, we will assume $\mathbb{k}$ to be algebraically closed of characteristic zero. Let $I$ be $J$-cellular for $J \subset[n]$ and let $\mathfrak{m}_{J}:=\left\langle x_{i}: i \notin J\right\rangle$. The associated primes of $I$ are of the form $I_{\rho, J}+\mathfrak{m}_{J}$ where $I_{\rho, J}:=\left\langle x^{u}-\rho(u-v) x^{v}: u-v \in L\right\rangle$ is a lattice ideal in the $J$-variables and $\rho: L \rightarrow \mathbb{k}^{*}$ is a group homomorphism from a sublattice $L \subset \mathbb{Z}^{J}$. The pair $(\rho, L)$ is called a partial character in [DMM,ES]. Here we will simply speak of a character. An extension of $\rho$ is a character $\tau: L^{\prime} \rightarrow \mathbb{k}$ 
such that $L \subset L^{\prime}$ and $\rho$ and $\tau$ agree on $L$. A character is saturated if its domain is a saturated lattice, that is $L$ is not contained with finite index in any other sublattice of $\mathbb{Z}^{J}$. An extension to a saturated character is a saturation. Set $\mathbb{k}[J]:=\mathbb{k}\left[x_{i}: i \in J\right]$ and $\mathbb{k}[\bar{J}]:=\mathbb{k}\left[x_{i}: i \notin J\right]$. Associated primes of cellular binomial ideals come in groups. The following theorem states that they are to be found among the associated primes of lattice ideals in $\mathbb{k}[J]$.

Theorem ([ES, Theorem 8.1]). Let $I \subset S$ be a J-cellular binomial ideal. Let $I_{\sigma, J}+\mathfrak{m}_{J}$ be an associated prime of $I$, then there exists a monomial $m \in \mathbb{k}[\bar{J}]$, and a character $\tau$ on $\mathbb{Z}^{J}$ whose saturation is $\sigma$, such that $(I: m) \cap \mathbb{k}[J]=I_{\tau}$.

The converse also holds. Every associated prime of any occurring lattice ideal is associated to $I$; see $[\mathrm{KM}]$. The Theorem shows that a sub-problem in the computation of associated primes is to determine the set of lattice ideals of the form $(I: m) \cap \mathbb{k}[J]$.

Let $I \subset S$ be a $J$-cellular binomial ideal. A lattice $L \subset \mathbb{Z}^{J}$ is associated to $I$ if there exists a witness monomial $m \in \mathbb{k}[\bar{J}]$ such that $(I: m) \cap \mathbb{k}[J]=I_{\rho, J}$ for some character $\rho: L \rightarrow \mathbb{k}^{*}$. This definition is a special case of [KM, Definition 12.1] which covers the case of an arbitrary binomial ideal in a monoid algebra. The lattice ideals $(I: m) \cap \mathbb{k}[J]$ are partially ordered by inclusion, and so are their lattices. An associated lattice is embedded, if it properly contains the lattice of $I \cap \mathbb{k}[J]$.

A first algorithm to find associated lattices would examine all ideals $(I: m)$ where $m$ is a nonzero monomial in $\mathbb{k}[\bar{J}] /(I \cap \mathbb{k}[\bar{J}])$. By cellularity of $I$, there are only finitely many such monomials and this search will terminate. The associated primes algorithm in Binomials instead uses a random search. The set of monomials to be examined can be very large compared to relatively few associated lattices. The design goal in the algorithm below is to compute as few colon ideals $(I: m)$ as possible. If a monomial $m$ divides a monomial $n$, then $(I: m) \subset(I: n)$ and containment also holds for the associated lattices. Due to this fact, we can exclude large posets of monomials if we find two monomials with the same associated lattice.

\section{Algorithm.}

Input: A $J$-cellular binomial ideal $I$.

Output: The associated lattices of $I$

(1) Compute the lattice ideal $I \cap \mathbb{k}[J]$.

(2) Initialize a list of known associated lattices and witnesses containing only the pair $(I \cap \mathbb{k}[J], 1)$.

(3) Initialize a todo-list with all monomials in a $\mathbb{k}$-basis of $\mathbb{k}[\bar{J}] /(I \cap \mathbb{k}[\bar{J}])$.

(4) Iterate the following until the todo-list is empty

- Choose and remove a random monomial $m$ from the todo-list. Compute the lattice ideal $(I: m) \cap \mathbb{k}[J]$ and check if its lattice is already on the list of associated lattices.

- If yes, then add $m$ as a new witness for that lattice, remove from the todo-list every monomial between existing witnesses and $m$.

- If no, then add $(I \cap \mathbb{k}[J], m)$ to the list of associated lattices.

To save space and time, the implementation in Binomials does not save all the witness monomials. If $m, n$ are both witnesses for the same associated lattice and $m \mid n$, then only $m$ needs to be saved.

Given the set of associated lattices, determining the associated primes is easy. It consists of saturating characters and will not be discussed here. The necessary cyclotomic extensions are handled in a separate package Cyclotomic published together with Binomials. 
COMPUTING MINIMAL PRIMARY COMPONENTS. Let $I \subset S$ be a $J$-cellular binomial ideal and let $P=I_{\rho, J}+\mathfrak{m}_{J}$ be one of its associated primes. Eisenbud and Sturmfels show that any primary component of $I$ over $P$ contains $I_{\rho, J}$. In fact, $I+I_{\rho, J}$ has $P$ as its unique minimal prime and a primary component is computed by removing all embedded primary components from $I+I_{\rho, J}$. This is the content of [ES, Theorem 7.1]. Let Hull $(I)$ denote the intersection of the minimal primary components of a binomial ideal $I$. If $I$ is cellular, then $\operatorname{Hull}(I)$ is binomial. Computing the hull of a binomial ideal is a cumbersome procedure. One way, described in [ES], is to successively identify binomials $b$ such that $(I: b)$ is a binomial ideal strictly containing $I$. This approach is slow. Here we will use a strategy similar to the one in the Algorithm. Denote $M_{\mathrm{emb}}(I)$ the monomial ideal generated by all witnesses of embedded lattices of $I$. Then [DMM, Theorem 3.2] implies the following simplification.

Proposition. If I is $J$-cellular and has exactly one minimal prime, then $\operatorname{Hull}(I)=I+M_{\mathrm{emb}}(I)$. In particular, Hull $(I)$ is binomial.

To compute the minimal primary component of $I$ over $P=I_{\rho, J}+\mathfrak{m}_{J}$, one computes $\operatorname{Hull}\left(I+I_{\rho, J}\right)$, see [ES, OS]. The monomial ideal $M_{\mathrm{emb}}\left(I+I_{\rho, J}\right)$ is determined essentially by the algorithm above. It is in fact simpler, since only minimal generators of $M_{\mathrm{emb}}(I)$ need to be computed. In most cases, only a small fraction of the standard monomials needs to be examined.

The ideal in the following example is cellular.

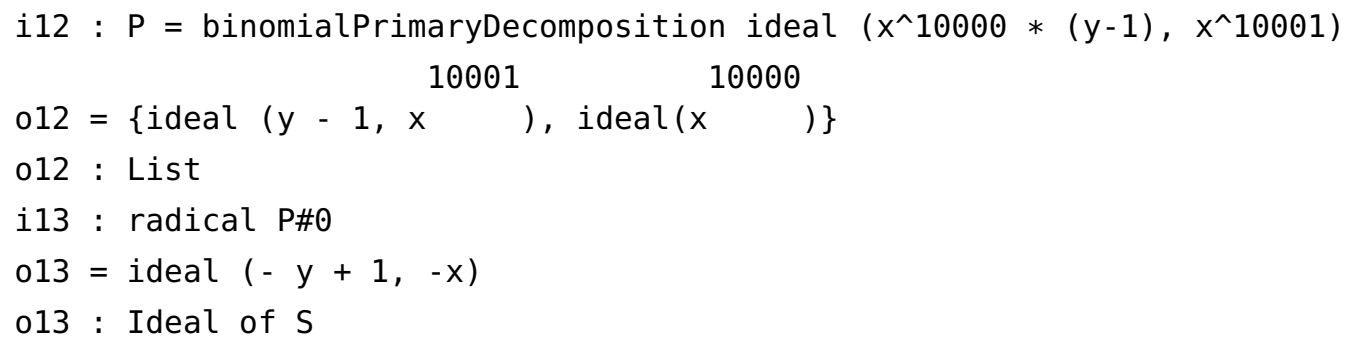

The embedded component over $\langle x, y-1\rangle$ is of high multiplicity. To find its embedded prime using the Theorem above, a-priori 10000 nilpotent monomials would have to be examined. In contrast, a typical run of the Algorithm above would only compute $\left\lceil\log _{2}(10000)\right\rceil=14$ lattice ideals. In this simple example, the nilpotent monomials are totally ordered, but in general they form complicated posets; see [KM, Problem 17.4]. It is not known if there are better search algorithms than the random search.

ACKNOWLEDGMENT. The author thanks the organizers and participants of the Macaulay 2 workshop March 2011 in Göttingen during which the improved algorithms were first implemented.

\section{REFERENCES.}

[DMM] A. Dickenstein, L.F. Matusevich, and E. Miller, Combinatorics of binomial primary decomposition, Math. Z. 264 (2010), no. 4, $745-763$.

[ES] D. Eisenbud and B. Sturmfels, Binomial ideals, Duke Math. J. 84 (1996), no. 1, 1 - 45.

[F] A. Fink, The binomial ideal of the intersection axiom for conditional probabilities, J. Algebraic Combin. 33 (2011), no. 3, $455-463$.

[M2] D.R. Grayson and M.E. Stillman, Macaulay2, a software system for research in algebraic geometry, available at www. math. uiuc. edu/Macaulay2/.

$\left[\mathrm{HHH}^{+}\right]$J. Herzog, T. Hibi, F. Hreinsdóttir, T. Kahle, and J. Rauh, Binomial edge ideals and conditional independence statements, Adv. in Appl. Math. 45 (2010), no. 3, 317 - 333. 
[K] T. Kahle, Decompositions of binomial ideals, Ann. Inst. Statist. Math. 62 (2010), no. 4, 727 - 745.

[KM] T. Kahle and E. Miller, Decompositions of commutative monoid congruences and binomial ideals, available at arXiv:1107.4699v3 [math. AC].

[OS] I. Ojeda Martínez de Castilla and R.P. Sánchez, Cellular binomial ideals. Primary decomposition of binomial ideals, J. Symbolic Comput. 30 (2000), no. 4, 383 - 400.

RECEIVED : 2011-06-28 REVISED : 2011-09-22 ACCEPTED : 2012-02-06

thomas . kahle@math . ethz.ch : ETH Zürch, Forschungsinstitut Mathematik, Office HG G 38.1, Rämistrasse 101, CH-8092 Zürich, Switzerland. 